\title{
Evaluation of true interlamellar spacing from microstructural observations
}

\author{
Teruyuki Ikeda \\ Materials Science, California Institute of Technology, Pasadena, California 91125 \\ Vilupanur A. Ravi \\ Department of Chemical and Materials Engineering, California State Polytechnic University, \\ Pomona, California 91768 \\ G. Jeffrey Snyder ${ }^{\text {a) }}$ \\ Materials Science, California Institute of Technology, Pasadena, California 91125
}

(Received 22 February 2008; accepted 19 June 2008)

A method for evaluating true interlamellar spacing from micrographs is proposed for a multidomained lamellar structure. The microstructure of these materials is assumed to be composed of many domains with the lamellae aligned roughly parallel to each other within each domain and with the domains themselves randomly oriented relative to one another. An explicit expression for the distribution of apparent interlamellar spacing is derived assuming that the distribution of the true interlamellar spacing is Gaussian. The average interlamellar spacing is close to the peak interlamellar spacing observed in the distribution. The theoretical distributions are compared with experimental ones obtained by analyzing micrographs of $\mathrm{PbTe}-\mathrm{Sb}_{2} \mathrm{Te}_{3}$ lamellar composites.

\section{INTRODUCTION}

Studies on the microstructures of alloys continue to be crucial because of their significant effect on functional and structural properties in many cases. Alloys that have lamellar structures, e.g., pearlitic steel, are in practical use and have been studied extensively. Interlamellar spacing is an important attribute of such lamellar structures. It is therefore essential to quantify this microstructural feature. For this purpose, the analysis on micrographs such as scanning electron microscopic or optical microscopic images is the simplest and the most direct method. In the analysis of micrographs, it is important to note the fact that the interlamellar spacings in the micrographs are not the true spacings because the lamellae are not necessarily oriented perpendicular to the observation planes. If the microstructure in the sample is oriented in a particular direction, e.g., because it has been unidirectionally solidified, the true interlamellar spacing can be determined by a procedure proposed by Bansal, ${ }^{1}$ where micrographs on two sections for each lamellar domain are required. However, it is not realistic to use this method for samples that have microstructures with randomly oriented polydomains. For materials with polydomains, Pellisier et al. ${ }^{2}$ have derived the expression for the

\footnotetext{
a) Address all correspondence to this author.

e-mail: jsnyder@caltech.edu

DOI: $10.1557 / J M R .2008 .0308$
}

distributions of apparent interlamellar spacing in terms of the distribution of the angle between lamellae and the observation plane. Furthermore, since true interlamellar spacing itself is not constant in most cases, methods to determine the distribution of the true interlamellar spacing from the cumulative area have been developed by several workers. ${ }^{2-5}$ In these methods, the true interlamellar spacing within each domain was assumed to be constant; however, there could be a distribution of true interlamellar spacing within domains as well as the distribution of average values of true interlamellar spacing of domains in the actual lamellar structure. Cahn and Full$\operatorname{man}^{6}$ proposed an analysis to obtain the distributions of true lamellar thickness from those in arbitrary directions on observation planes. Their method is equally applicable to the analysis of lamellar spacings. However, as we show in this work, the method of Cahn and Fullman is sensitive to noise and requires a large number of observations. This is due to a derivative term in their analysis, as discussed later. All of the works mentioned above motivated the authors to develop a simple but reasonable method for practical use.

In the present work, we have derived an expression for the distribution of the apparent interlamellar spacing taking into account the distribution of the angle between lamellae and the observation plane and the distribution of true interlamellar spacing without assuming constant spacing in domains. To derive the explicit form of the expression, we used an assumption that the true lamellar 
spacing obeys a Gaussian distribution. Then, the theoretical equation of the distribution of interlamellar spacing was compared with that obtained experimentally by analyzing scanning electron images of a $\mathrm{PbTe}-\mathrm{Sb}_{2} \mathrm{Te}_{3}$ alloy. This system is of particular interest because a lamellar structure consisting of $\mathrm{PbTe}$ and $\mathrm{Sb}_{2} \mathrm{Te}_{3}$ is obtained by the decomposition of the metastable compound $\mathrm{Pb}_{2} \mathrm{Sb}_{6} \mathrm{Te}_{11}$ upon annealing. ${ }^{7}$ The present method was also compared with the one proposed by Cahn and Fullman, ${ }^{6}$ which converts the distribution of spacings measured in arbitrary directions to that of true spacings.

\section{THE MODEL}

In this work, we assume that the microstructure of a sample is composed of many domains with the lamellae aligned roughly parallel to one another within each domain and with the domains themselves randomly oriented relative to one another. The sample is arbitrarily sliced to investigate the microstructure in a plane, denoted the observation plane. Figure 1 shows a schematic defining the different spacings considered in this paper. From this observation plane, the spacing between the observed lamellae is measured along an observation line perpendicular to the observed lamellar interfaces. The observed lamellar spacing (full period of light-dark variation) is then measured at each full period along the entire observation line for each domain. For each measurement point, the observed spacing $S$ (length) may be larger than the true spacing $S_{0}$ of the layers because the observation plane is not necessarily normal to the plane of the lamellae at the observation point. Here we define the angle between the observation plane and the lamellar plane as $\theta$. To derive the relationship between the distri-

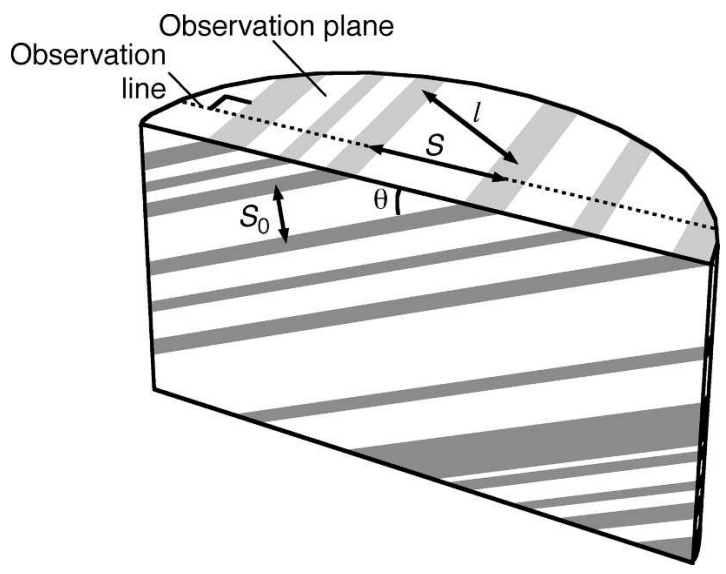

FIG. 1. Definition of the angle between a normal vector of lamellae and observation section $\theta$, true lamellar spacing $S_{0}$, apparent lamellar spacing $S$. An observation plane is randomly oriented with respect to the lamellae. The lateral plane where $S_{0}$ and $\theta$ are defined is perpendicular to both the observation plane and the lamellae. $S$ and $S_{0}$ are measured along lines perpendicular to lamellae on each plane. $l$ is the random spacing used in the analysis by Cahn and Fullmann. ${ }^{6}$ bution of observed spacings $S$ and the true interlamellar spacing $S_{0}$, the following assumptions were used:

(i) The sample to be analyzed has a polydomain structure with many randomly oriented domains, i.e., $\theta$ can assume random values.

(ii) The distribution of the volume fraction of the region with the true interlamellar spacing $S_{0}$ obeys a Gaussian distribution against the variation of $S_{0}$.

As shown by Pellissier, ${ }^{2}$ the probability that the spacing of a given domain on the observation plane lies between $S$ and $S+\mathrm{d} S$ is given by $P_{\mathrm{S}} \mathrm{d} S$, where $P_{\mathrm{S}}$ is the probability density function expressed as

$$
P_{\mathrm{S}}=\frac{S_{0}{ }^{2}}{S^{2} \sqrt{S^{2}-S_{0}^{2}}},
$$

taking into account assumption (i) mentioned above. According to assumption (ii), the volume fraction of the region where the true interlamellar spacing is in the range between $S_{0}$ and $S_{0}+\mathrm{d} S_{0}$ obeys a Gaussian distribution $P_{\mathrm{S}_{0}} \mathrm{~d} S_{0}$, where $P_{\mathrm{S}_{0}}$ is the probability density function given by

$$
P_{\mathrm{S}_{0}}=\frac{1}{\sqrt{2 \pi} \sigma} e^{\frac{\left(S_{0}-S_{0, \mathrm{ave}}\right)^{2}}{2 \sigma^{2}}},
$$

where $S_{0, \text { ave }}$ and $\sigma$ are the average and the standard deviation of true lamellar spacing, respectively. While both of these probabilities $P_{\mathrm{S}} \mathrm{d} S$ and $P_{\mathrm{S}_{0}} \mathrm{~d} S_{0}$ were derived or used with respect to volume fractions, they should be equally valid as the probabilities with respect to the length fractions of the observation line.

In our observations, we recorded the spacing of each observed lamellae as opposed to the average value of each domain. ${ }^{3-5}$ We must take into consideration that larger apparent spacings will result in fewer number of observations compared to smaller apparent spacings. Within the total length of the observation line in a sample, the fraction of the line that lies on the region where true lamellar spacing is between $S_{0}$ and $S_{0}+\mathrm{d} S_{0}$, and apparent spacing is between $S$ and $S+\mathrm{d} S$ is $P_{\mathrm{S}} P_{\mathrm{S}_{0}} \mathrm{~d} S \mathrm{~d} S_{0}$. For lamellar spacing $S$, the number of lamellae observed per unit length is $1 / S$. Thus, following the way of relating the lineal fraction to the number of intercepts per unit length by Cahn and Fullman in their analysis on platelet thickness distribution, ${ }^{6}$ the number of lamellae that have the true lamellar spacing from $S_{0}$ to $S_{0}+\mathrm{d} S_{0}$ and the apparent lamellar spacing from $S$ to $S+$ $\mathrm{d} S$ observed per unit length of observation line is expressed by the distribution,

$$
N S_{0} S \mathrm{~d} S_{0} \mathrm{~d} S=\frac{P_{\mathrm{S}_{0}} P S}{S} \mathrm{~d} S_{0} \mathrm{~d} S
$$

The number of lamellae that have the apparent lamellar spacing from $S$ to $S+\mathrm{d} S$ per unit length of observation 
line $N_{\mathrm{S}} \mathrm{d} S$ is obtained by integration of Eq. (3) with respect to $S_{0}$ in the range from 0 to $S$,

$$
\begin{aligned}
& N_{\mathrm{S}} \mathrm{d} S=\left(\int_{0}^{S} N_{S_{0} S} \mathrm{~d} S_{0}\right) \mathrm{d} S= \\
& \frac{1}{S^{3} \sqrt{2 \pi} \sigma}\left(\int_{0}^{S} e^{-\frac{\left(S_{0}-S_{0, \text { ave }}\right)^{2}}{2 \sigma^{2}}} \frac{S_{0}^{2}}{\sqrt{S^{2}-S_{0}^{2}}} \mathrm{~d} S_{0}\right) \mathrm{d} S .
\end{aligned}
$$

This equation can now be used to draw a theoretical distribution of apparent lamellar spacing with input parameters $S_{0 \text {,ave }}$ and $\sigma$. Subsequently, one can determine $S_{0, \text { ave }}$ and $\sigma$ by comparing the theoretical distribution with the experimental one. Because the apparent total number of lamellae per unit of observation length is obtained by integration of Eq. (4) with respect to $S$ from 0 to $\infty$, it would be more convenient to use the fraction of lamellae that have apparent lamellar spacings between $S_{1}$ and $S_{2}$ to the total number of lamellae expressed as

$$
\begin{aligned}
F\left(S_{1}, S_{2}\right)= & \frac{\int_{S_{1}}^{S_{2}} N_{\mathrm{S}} \mathrm{d} S}{\int_{0}^{\infty} N_{\mathrm{S}} \mathrm{d} S} \\
= & \frac{\int_{S_{1}}^{S_{2}} \frac{1}{S^{3}}\left(\int_{0}^{S} e^{-\frac{\left(S_{0}-S_{0, \text { ave }}\right)^{2}}{2 \sigma^{2}}} \frac{S_{0}^{2}}{\sqrt{S^{2}-S_{0}^{2}}} \mathrm{~d} S_{0}\right) \mathrm{d} S}{\int_{0}^{\infty} \frac{1}{S^{3}}\left(\int_{0}^{S} e^{-\frac{\left(S_{0}-S_{0, \mathrm{ave}}\right)^{2}}{2 \sigma^{2}}} \frac{S_{0}{ }^{2}}{\sqrt{S^{2}-S_{0}^{2}}} \mathrm{~d} S_{0}\right) \mathrm{d} S}
\end{aligned}
$$

This equation does not require the measurement of the lengths of the observation line.

Figure 2 shows the distributions calculated for several values of $S_{0 \text {,ave }}$ and a constant $\sigma(=20 \mathrm{~nm})$. The tails of the peaks are longer for the larger lamellar spacings because they are affected by the distribution of domain orientation in addition to the distribution of true interlamellar spacing, whereas for the smaller spacings, the tails are sensitive only to the distribution of true interlamellar spacing. Figure 3 shows the distributions drawn with several values of $\sigma$ and a constant $S_{0, \text { ave }}(=500 \mathrm{~nm})$. The tails of peaks are longer for the larger $\sigma$ as expected.

The peak position of the distribution is found to be close to the true average value $S_{0 \text {,ave }}$, suggesting the peak position could be simply used for routine analysis. The peak position of the distribution of $S$ can be determined as a solution to the equation $\mathrm{d} N_{\mathrm{S}} / \mathrm{d} S=0$. Introducing a new variable $\varphi$ for mathematical convenience, $S_{0}=S$ $\sin \varphi$ and $r=\sigma / S$ and $r_{0, \text { ave }}=\sigma / S_{0, \text { ave }}$, gives

$$
r \frac{\mathrm{d} f}{\mathrm{~d} r}+f=0
$$

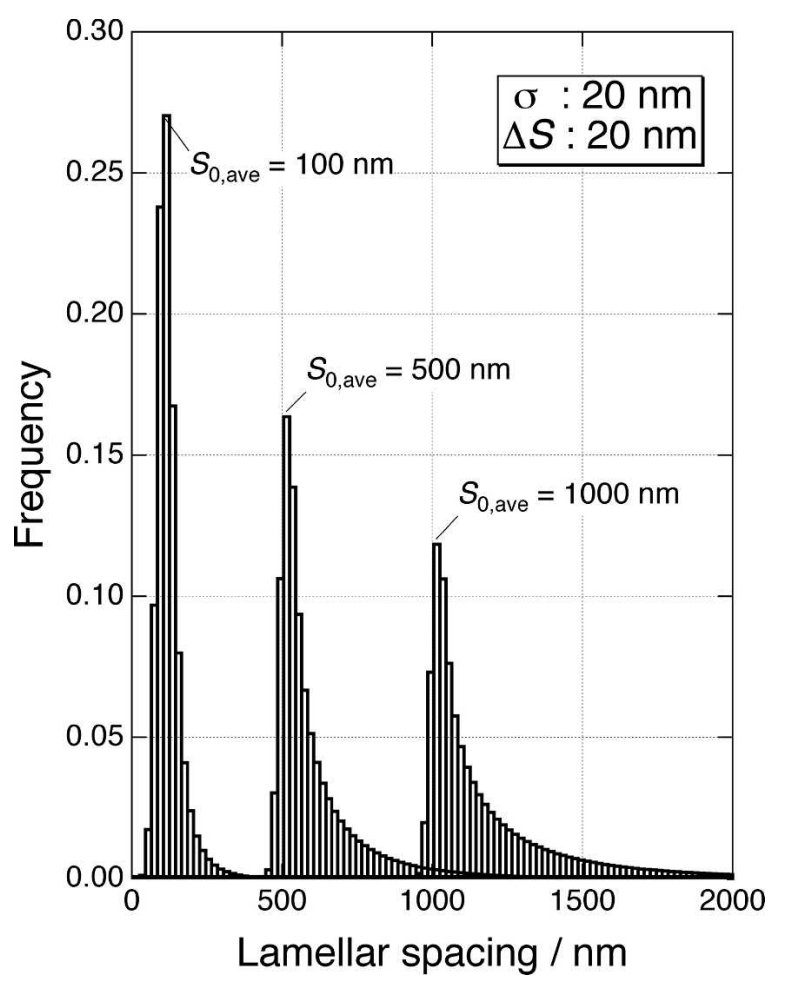

FIG. 2. Frequencies of apparent lamellar spacing calculated using Eq. (5) with several values of $S_{0, \text { ave }}$ and a constant $\sigma(=20 \mathrm{~nm})$. The column width $\Delta S$ was taken to be $20 \mathrm{~nm}$.

where

$$
f=\int_{0}^{\pi} \frac{\pi}{2} e^{-\frac{1}{2}\left(\frac{1}{r} \sin \varphi-\frac{1}{r_{0, \text { ave }}}\right)^{2}} \sin ^{2} \varphi \mathrm{d} \varphi .
$$

Because the left-hand side of Eq. (6a) is a function of only $r$ for a given $r_{0 \text {, ave, }}$, the peak position $r_{\text {peak }}(=$ $\left.\sigma / S_{\text {peak }}\right)$ is determined as a function of $r_{0, \text { ave }}$. Figure 4 shows the peak position $S_{\text {peak }} / S_{0 \text {,ave }}\left(=r_{0, \text { ave }} / r_{\text {peak }}\right)$ as a function of $\sigma / S_{0 \text {,ave }}\left(=r_{0 \text {,ave }}\right)$. In the range of $r_{0 \text {,ave }}$ shown in the graph, $S_{\text {peak }} / S_{0, \text { ave }}$ is mostly within $1 \pm 0.05$. This means the difference between $S_{\text {peak }}$ and $S_{0, \text { ave }}$ is smaller than the bin size $(\Delta S)$ in all cases shown in Figs. 2 and 3 . In the region with high values of $\sigma / S_{0 \text {,ave }}$ more than $\sim 0.1, S_{\text {peak }} / S_{0 \text {,ave }}$ decreases eventually to values less than 1 . This is because the factor $1 / S^{3}$ in Eq. (5) diverges for $S \approx 0$, which shifts $S_{\text {peak }}$ to lower values. However, $S \approx 0$ in Eq. (5) is nonphysical because there should be a lower limit to the size of the features (at least one unit cell). Thus, the model breaks down for $\sigma / S_{0, \text { ave }} \gtrsim 0.3$.

\section{COMPARISON WITH EXPERIMENTAL RESULTS}

A sample of $\mathrm{Pb}_{10.5} \mathrm{Sb}_{31.5} \mathrm{Te}_{58}$ alloy was prepared by solidification processing. The details of the preparation method are reported elsewhere. ${ }^{7}$ A small piece of about $5 \times 5 \mathrm{~mm}$ was annealed at $773 \mathrm{~K}$ for $4.32 \times 10^{5} \mathrm{~s}$ ( 5 days) under vacuum in a fused silica tube. The surface prepa- 

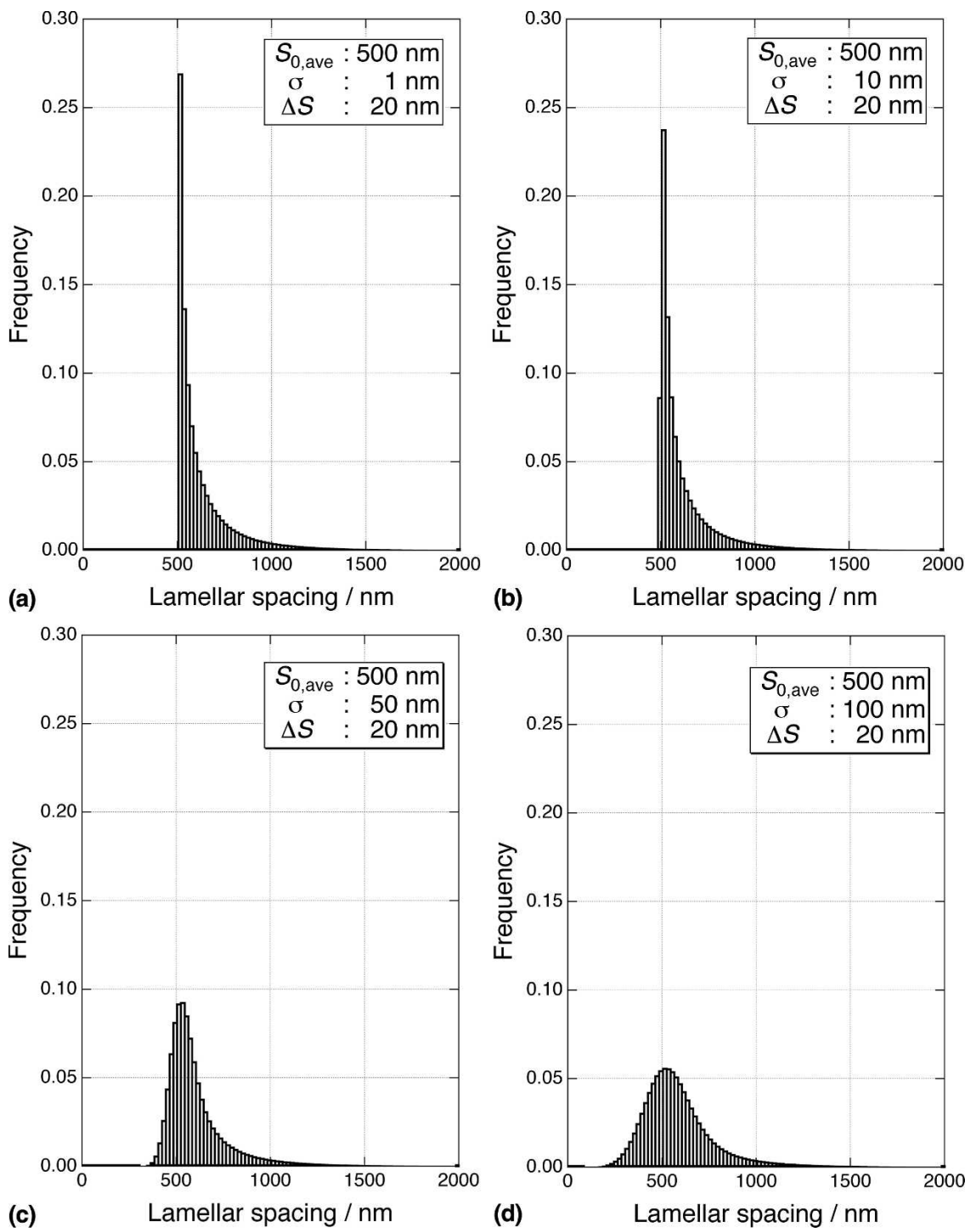

FIG. 3. Frequencies of apparent lamellar spacing calculated using Eq. (5) with a constant $S_{0, \text { ave }}(=500 \mathrm{~nm})$ and values of $\sigma$ of (a) $1 \mathrm{~nm}$, (b) $10 \mathrm{~nm}$, (c) $50 \mathrm{~nm}$, and (d) $100 \mathrm{~nm} . \Delta S$ was taken to be $20 \mathrm{~nm}$.

ration procedure for microstructural observation by scanning electron microscopy and the observation condition were also described elsewhere. ${ }^{7}$ Figure 5 shows an example of the microstructure obtained by the backscatter electron mode. The microstructure is composed of $\mathrm{PbTe}+\mathrm{Sb}_{2} \mathrm{Te}_{3}$ lamellae, which form by the decomposition of $\mathrm{Pb}_{2} \mathrm{Sb}_{6} \mathrm{Te}_{11}$, and random regions of the two phases. Note the alignment of the lamellae in the domains. The backscattered electron images were digitally analyzed to determine interlamellar spacing using image analysis software (Macscope, Mitani Corp., Tokyo, Japan). Eight micrographs were obtained from randomly selected regions across the sample. Each micrograph used in the analysis typically contained 400-500 lamellae. The spacing between adjacent pairs of $\mathrm{PbTe}+$ $\mathrm{Sb}_{2} \mathrm{Te}_{3}$ lamellae was measured for the entire field of view in each micrograph.
Figure 6 shows the distribution of interlamellar spacings, i.e., the apparent interlamellar spacings, obtained by the image analysis mentioned above. The distributions of interlamellar spacing from eight images were summed to obtain the total distribution. The tail is longer for the larger interlamellar spacing, an observation consistent with the theoretical distributions described earlier. In Fig. 6, a theoretical distribution using Eq. (5) fitted to the experimental data by the least square method is also shown. The experimental distribution agrees fairly well with the theoretical distribution. In this analysis, the average value $S_{0, \text { ave }}$ and the standard deviation $\sigma$ of the true interlamellar spacing were determined to be 910 and $212 \mathrm{~nm}$, respectively, as fitting parameters. The solid circles represent the Gaussian distribution of the volume fraction of the regions that have the true lamellar spacing $S_{0}$ drawn using the above parameters. 


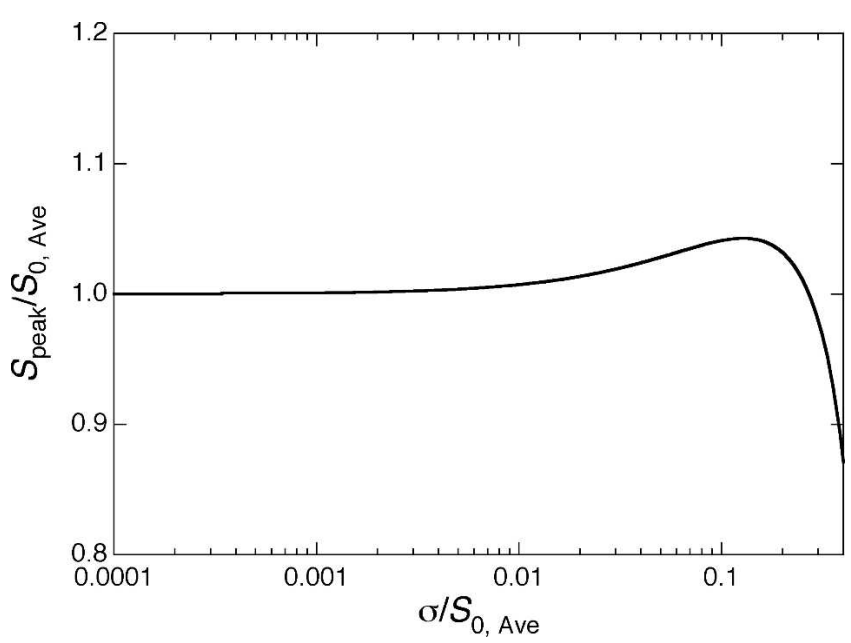

FIG. 4. Ratio of the apparent lamellar spacing at the peak in the distribution $S_{\text {peak }}$ to the average true lamellar spacing $S_{0 \text {,ave }}$, calculated using Eq. (6a) as a function of $\sigma / S_{0, \text { ave }}$.

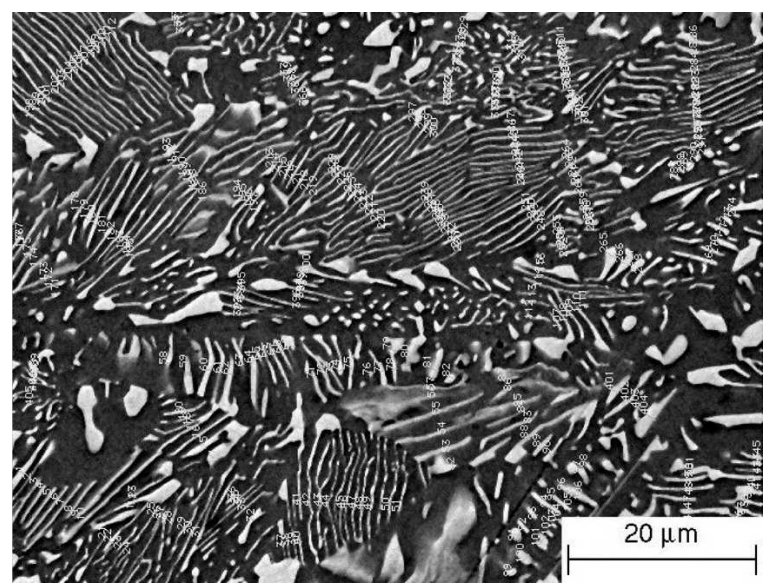

FIG. 5. Part of a backscatter electron image of a $\mathrm{Pb}_{10.5} \mathrm{Sb}_{31.5} \mathrm{Te}_{58}$ alloy after annealing at $773 \mathrm{~K}$ for $4.32 \times 10^{5} \mathrm{~s}$ ( 5 days). A bright phase is $\mathrm{PbTe}$ and a dark phase is $\mathrm{Sb}_{2} \mathrm{Te}_{3}$. The interlamellar spacings have been measured along to the lines shown in the picture.

For this sample, the experimental distribution of apparent interlamellar spacing is reproduced well by the theoretical one. This means the actual distribution of the true spacing is nearly Gaussian. In general, however, the distribution of true lamellar spacing in the microstructure obtained by nucleation and growth process might be dependent on the distribution at the beginning of the transformation, the growth mechanism of the transformed region, and/or the coarsening process, and hence may not necessarily be Gaussian.

\section{DISCUSSION}

In the method described here and those used in previous works, ${ }^{2-5}$ the operator analyzing the images must define domain boundaries and select the perpendicular observation line for each domain. This leads to a possible

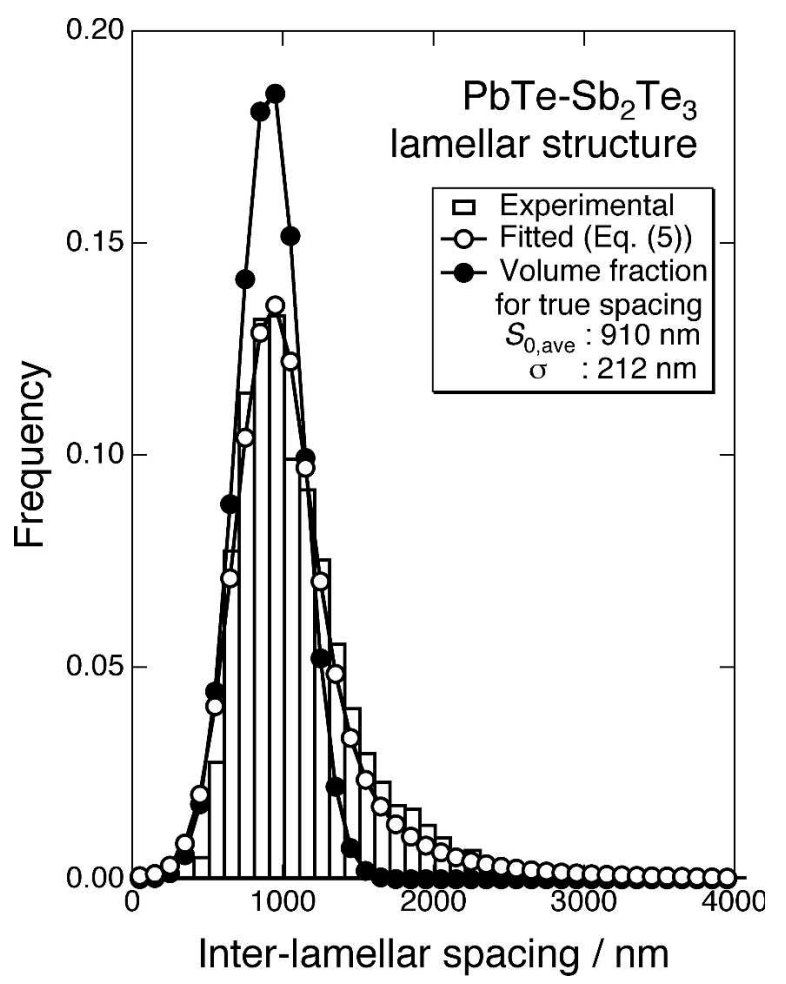

FIG. 6. Experimental (bars) and theoretical (open circles and lines) distribution of the apparent interlamellar spacing in $\mathrm{a} \mathrm{Pb}_{10.5} \mathrm{Sb}_{31.5} \mathrm{Te}_{58}$ alloy after annealing at $773 \mathrm{~K}$ for $4.32 \times 10^{5} \mathrm{~s}$ ( 5 days). The theoretical distribution is the best fitted to the experimental one with the parameters $S_{0, \text { ave }}=910 \mathrm{~nm}$ and $\sigma=212 \mathrm{~nm}$ using Eq. (5). Solid circles show the Gaussian distribution for the volume fraction of the regions that have true lamellar spacing $S_{0}$ drawn using the parameters $S_{0 \text {,ave }}=$ $910 \mathrm{~nm}$ and $\sigma=212 \mathrm{~nm}$.

operator effect that may skew the results in favor of particular domain types or orientations. The method of Cahn and Fullman, ${ }^{6}$ however, is much more operator independent and therefore potentially better suited for such observations.

Cahn and Fullman ${ }^{6}$ analyzed the spacings observed not only in a random observation plane but also from a randomly oriented observation line that is not necessarily perpendicular to the observed lamellae. (While, in their paper, ${ }^{6}$ an equation to convert the distribution of the lamellar thickness in random directions to that of the true lamellar thickness are shown, the same equation can be used for the conversion of the interlamellar spacings.) Using their analysis, it is possible to determine the distribution of true interlamellar spacing from the distribution of observed lamellar spacing $l$ along arbitrarily oriented directions. While their analysis to determine the distribution of true domain size has been successfully used in many investigations (for example, Ref. 8), the analysis on the true lamellar spacing has not been used by many researchers. To compare the usefulness of the present method and that of Cahn and Fullman, analyses using both methods also carried out on the same micrographs. The method of Cahn and Fullman is easier to 
implement because the observation line need not be perpendicular to the lamellae in contrast to the method described above. Figure 7 shows an example of micrograph analyzed, which is on the same micrograph as the above mentioned method (Fig. 5). Spacings were measured along grids with $10-\mu \mathrm{m}$ intervals, which correspond to the line length of around $2000 \mu \mathrm{m}$ in total, on each micrograph. Using this method, 5268 spacings were recorded (Fig. 8), compared to the 2371 spacings recorded using the previously described method. The tail for larger spacings is longer than that in the distribution in Fig. 6 because of the additional degree of misalignment. Figure 8 also shows the distribution of the true interlamellar spacing as determined using Eq. (7) from Cahn and Fullman:

$$
V\left(S_{0}\right)_{S_{0}=l}=3 \operatorname{lm}(l)+l^{2} \frac{\mathrm{d} m(l)}{\mathrm{d} l},
$$

where $S_{0}$ is true interlamellar spacing, $l$ is lamellar spacing along randomly oriented measurement direction, $V\left(S_{0}\right)$ is the fraction of the volume with the true interlamellar spacing $S_{0}$, and $m(l) \mathrm{d} l$ is the number of spacings per unit length with spacing $l$ to $l+\mathrm{d} l$ in random directions. We might expect the true interlamellar spacing determined by the conversion of the experimental distribution using Cahn and Fullman's method to show a shoulder or second broad peak. This is because the image contains regions that do not have lamellar structure, such as those formed during solidification as opposed to annealing ${ }^{7}$ or have been coarsened resulting in globular structure or are located at the interface between neighboring domains. Such regions were excluded in the analysis by the method described in this paper and used in the analysis on annealed samples. ${ }^{7}$ However, the distribution derived from Cahn and Fullman contains too much noise to see such additional features clearly. The

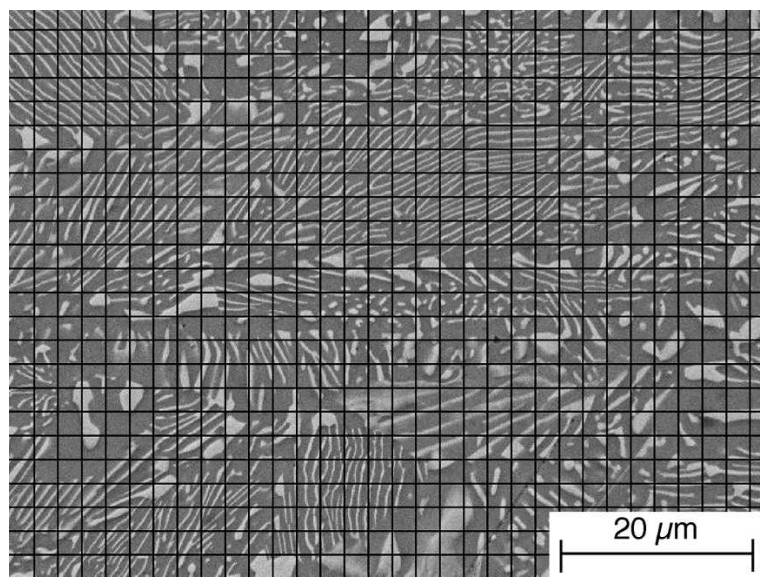

FIG. 7. Part of a backscatter electron image of a $\mathrm{Pb}_{10.5} \mathrm{Sb}_{31.5} \mathrm{Te}_{58}$ alloy after annealing at $773 \mathrm{~K}$ for $4.32 \times 10^{5} \mathrm{~s}$ ( 5 days), which is the same micrograph as Fig. 5 but with grid for analysis by Cahn and Fullman's method.

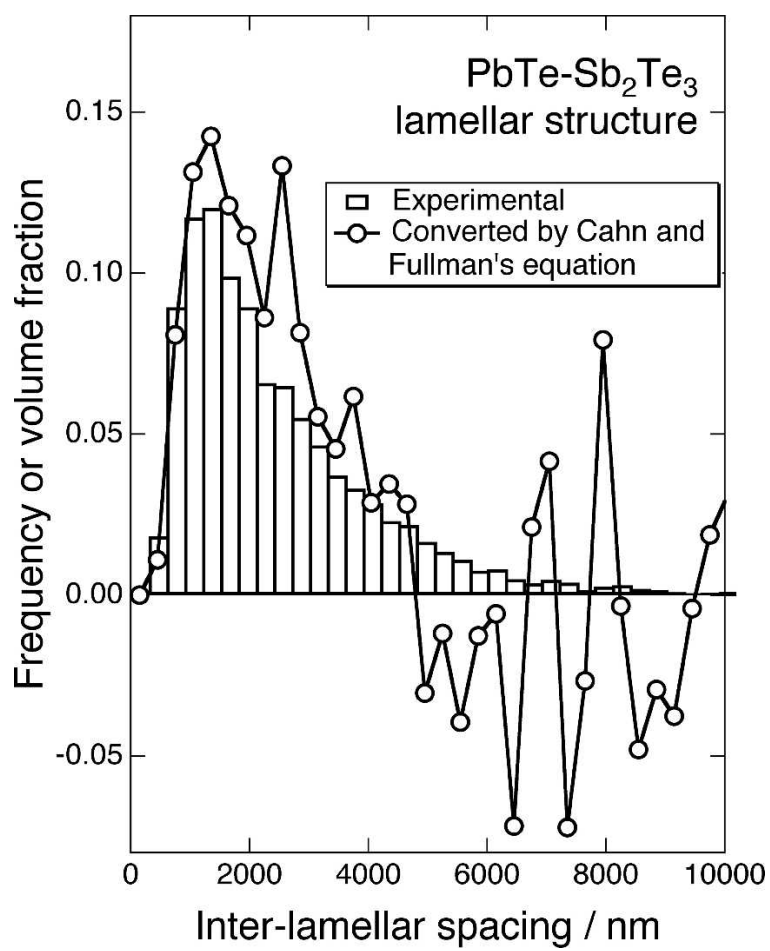

FIG. 8. Frequency of interlamellar spacing in random directions (bars) and the volume fraction of the domains plotted versus the true interlamellar spacing (circles and lines) converted from the spacing in random directions using Cahn and Fullman's equation [Eq. (7)] in a $\mathrm{Pb}_{10.5} \mathrm{Sb}_{31.5} \mathrm{Te}_{58}$ alloy after annealing at $773 \mathrm{~K}$ for $4.32 \times 10^{5} \mathrm{~s}$ (5 days).

sensitivity to noise using Cahn and Fullman's method is due to the derivative term in Eq. (7). The noise overwhelms the signal above about $5000 \mathrm{~nm}$ interlamellar spacing. Nevertheless, the resultant distribution of interlamellar spacing in Fig. 8 is noticeably asymmetric (not Gaussian) with a sharper reduction in interlamellar spacing below the average and broader decrease for interlamellar spacing above the average. Moreover, the converted distribution derived from Eq. (7) peaks at a little larger spacing $(1350 \mathrm{~nm})$ than that found with the other method $(950 \mathrm{~nm})$. These features are attributed to spacing from non-lamellar regions mentioned above.

\section{CONCLUSIONS}

We have demonstrated a method useful for the determination of an average value $S_{0 \text {,ave }}$ and a standard deviation $\sigma$ of true interlamellar spacing from micrographs. The proposed method gives a reasonable fit to the observed distribution of interlamellar spacing in the $\mathrm{Sb}_{2} \mathrm{Te}_{3} / \mathrm{PbTe}$ lamellar structure and is useful for characterizing this material. The average interlamellar spacing is close to the peak interlamellar spacing observed in the distribution. In principle, the method of Cahn and Fullman should reveal the true distribution with less dependence on the operator performing the method, but its 
implementation requires a far greater number of observations for comparable resolution and the results include the spacing from domain boundary and non-lamellar regions. The present method is adequate to extract the distribution of spacings only from the regions with truly lamellar structure without noise from non-lamellar regions.

\section{ACKNOWLEDGMENT}

This work was funded by the National Aeronautics and Space Administration (NASA) through the Jet Propulsion Laboratory.

\section{REFERENCES}

1. V. Bansal: A procedure to determine true lamellar dendrite spacing. J. Mater. Sci. Lett. 8, 451 (1989).
2. G.E. Pellissier, M.F. Hawkes, W.A. Johnson, and R.F. Mehl: The interlamellar spacing of pearlite. Trans. ASM 30, 1049 (1942).

3. A. Roósz, Z. Gácsi, and M.K. Baan: A simple method for determining the true interlamellar spacing. Metallogr. 13, 299 (1980).

4. G.F. Vander Voort and A. Roósz: Measurement of the interlamellar spacing of pearlite. Metallogr. 17, 1 (1984).

5. A. Crarski and J. Rýs: Quantitative analysis of lamellar structure. Prace Komisji Metalurgiczno-Odlewniczej, Metallurgia 35, 35 (1988).

6. J.W. Cahn and R.L. Fullman: On the use of lineal analysis for obtaining particle size distribution functions in opaque samples. Trans. AIME 206, 610 (1956).

7. T. Ikeda, L.A. Collins, V.A. Ravi, F.S. Gascoin, S.M. Haile, and G.J. Snyder: Self-assembled nanometer lamellae of thermoelectric $\mathrm{PbTe}$ and $\mathrm{Sb}_{2} \mathrm{Te}_{3}$ with epitaxy-like interfaces. Chem. Mater. 19. 763 (2007)

8. A.G. Evans and T.G. Langdon: Structural ceramics. Prog. Mater. Sci. 21, 171 (1976). 